\title{
ON THE HASSE PRINCIPLE FOR FINITE GROUP SCHEMES OVER GLOBAL FUNCTION FIELDS
}

\author{
Cristian D. GonzÁlez-Avilés And Ki-Seng TAN
}

\begin{abstract}
Let $K$ be a global function field of characteristic $p>0$ and let $M$ be a (commutative) finite and flat $K$-group scheme. We show that the kernel of the canonical localization map $H^{1}(K, M) \longrightarrow \prod_{\text {all } v} H^{1}\left(K_{v}, M\right)$ in flat (fppf) cohomology can be computed solely in terms of Galois cohomology. We then give applications to the case where $M$ is the kernel of multiplication by $p^{m}$ on an abelian variety defined over $K$.
\end{abstract}

\section{Statement of the main theorem}

Let $K$ be a global field and let $\bar{K}$ be a fixed algebraic closure of $K$. Let $K^{\text {s }}$ be the separable closure of $K$ in $\bar{K}$ and set $G_{K}=\operatorname{Gal}\left(K^{\mathrm{s}} / K\right)$. Further, for each prime $v$ of $K$, let $\bar{K}_{v}$ be the completion of $\bar{K}$ at a fixed prime $\bar{v}$ of $\bar{K}$ lying above $v$ and let $K_{v}^{\text {s }}$ denote the completion of $K^{\mathrm{s}}$ at the prime of $K^{\mathrm{s}}$ lying below $\bar{v}$. Set $G_{v}=\operatorname{Gal}\left(K_{v}^{\mathrm{s}} / K_{v}\right)$. If $M$ is a commutative, finite and flat $K$-group scheme, let $H^{i}\left(G_{K}, M\right)$ (respectively, $H^{i}\left(G_{v}, M\right)$ ) denote the Galois cohomology group $H^{i}\left(G_{K}, M\left(K^{\mathrm{s}}\right)\right.$ ) (respectively, $\left.H^{i}\left(G_{v}, M\left(K_{v}^{\mathrm{s}}\right)\right)\right)$. The validity of the Hasse principle for $M\left(K^{\mathrm{s}}\right)$, i.e., the injectivity of the canonical localization map $H^{1}\left(G_{K}, M\right) \rightarrow \prod_{\text {all } v} H^{1}\left(G_{v}, M\right)$ in Galois cohomology, has been discussed in [6, 12]. See also [9, Section I.9, pp. 117-120]. However, if $K$ is a global function field of characteristic $p>0$ and $M$ has $p$-power order, the injectivity of the canonical localization map $\beta^{1}(K, M): H^{1}(K, M) \rightarrow \prod_{\text {all } v} H^{1}\left(K_{v}, M\right)$ in flat (fppf) cohomology has not been discussed before (but see [7, Lemma 1] for some particular cases). In this paper we investigate this problem and show that the injectivity of $\beta^{1}(K, M)$ depends only on the finite $G_{K^{-}}$-module $M\left(K^{\mathrm{s}}\right)$, which may be regarded as the maximal étale $K$-subgroup scheme of $M$. Indeed, let $\amalg^{1}(K, M)=$ $\operatorname{Ker} \beta^{1}(K, M)$ and set $\amalg^{1}\left(G_{K}, M\right)=\operatorname{Ker}\left[H^{1}\left(G_{K}, M\right) \rightarrow \prod_{\text {all } v} H^{1}\left(G_{v}, M\right)\right]$. Then the following holds.

Main theorem. Let $K$ be a global function field of characteristic $p>0$ and let $M$ be a commutative, finite and flat $K$-group scheme. Let $v$ be any prime of $K$. Then the inflation map $H^{1}\left(G_{K}, M\right) \rightarrow H^{1}(K, M)$ induces an isomorphism

$$
\operatorname{Ker}\left[H^{1}\left(G_{K}, M\right) \rightarrow H^{1}\left(G_{v}, M\right)\right] \simeq \operatorname{Ker}\left[H^{1}(K, M) \rightarrow H^{1}\left(K_{v}, M\right)\right] .
$$

In particular, $\amalg^{1}(K, M) \simeq \amalg^{1}\left(G_{K}, M\right)$.

Thus, the Hasse principle holds for $M$, i.e., $\amalg^{1}(K, M)=0$, if, and only if, the Hasse principle holds for the $G_{K}$-module $M\left(K^{\mathrm{s}}\right)$. An interesting example is the following. Let $A$ be an ordinary abelian variety over $K$ such that the Kodaira-Spencer map has

Received by the editors January 3, 2012.

2000 Mathematics Subject Classification. Primary 11G35; Secondary 14K15. 
maximal rank. Then $A_{p^{m}}\left(K^{\mathrm{s}}\right)=0$ for every integer $m \geq 1$ [17, Proposition, p.1093], and we conclude that the Hasse principle holds for $A_{p^{m}}$.

The theorem is proved in the next section. In Section 3 we develop some applications of the theorem and Section 4 contains some concluding remarks.

\section{Proof of the main theorem}

We keep the notation introduced in the previous section. In addition, we will write $(\operatorname{Spec} K)_{\mathrm{fl}}$ for the flat site on Spec $K$ as defined in [8, II.1, p.47], and $H^{r}(K, M)$ will denote $H^{r}\left((\operatorname{Spec} K)_{\mathrm{f}}, M\right)$. We will see presently that, in fact, $H^{r}(K, M) \simeq$ $H^{r}\left((\operatorname{Spec} K)_{\mathrm{fppf}}, M\right)$.

By a theorem of M. Raynaud (see [14] or [1], Theorem 3.1.1, p. 110), there exist abelian varieties $A$ and $B$ defined over $K$ and an exact sequence of $K$-group schemes

$$
0 \rightarrow M \stackrel{\iota}{\rightarrow} A \stackrel{\psi}{\rightarrow} B \rightarrow 0
$$

where $\iota$ is a closed immersion. For $r \geq 0$, let $\iota^{(r)}: H^{r}(K, M) \rightarrow H^{r}(K, A)$ and $\psi^{(r)}: H^{r}(K, A) \rightarrow H^{r}(K, B)$ be the maps induced by $\iota$ and $\psi$. The long exact flat cohomology sequence associated to (1) yields an exact sequence

$$
0 \rightarrow \operatorname{Coker} \psi^{(r-1)} \rightarrow H^{r}(K, M) \rightarrow \operatorname{Ker} \psi^{(r)} \rightarrow 0,
$$

where $r \geq 1$. Since the groups $H^{r}(K, A)$ and $H^{r}(K, B)$ coincide with the corresponding étale and fppf cohomology groups by [8, Theorem III.3.9, p.114] and [5, Theorem 11.7 , p.180], we conclude that $H^{r}(K, M)=H^{r}\left((\operatorname{Spec} K)_{\text {fppf }}, M\right)$.

Lemma 2.1. Let $v$ be any prime of $K$. If $A$ is an abelian variety defined over $K$, then $A\left(K^{\mathrm{s}}\right)=A(\bar{K}) \cap A\left(K_{v}^{\mathrm{s}}\right)$, where the intersection takes place inside $A\left(\bar{K}_{v}\right)$.

Proof. Let $F / K$ be a finite subextension of $K^{\mathrm{s}} / K$ and let $F_{v}$ denote the completion of $F$ at the prime of $F$ lying below $\bar{v}$. Choose an element $t \in F$ such that $F_{v}=k((t))$, where $k$ is the field of constants of $F$, and let $m \geq 1$ be an integer. Then $F_{v}^{p^{-m}}=$ $k\left(\left(t^{p^{-m}}\right)\right)=\sum_{i=1}^{p^{m}}\left(t^{p^{-m}}\right)^{i} F_{v} \subset F^{p^{-m}} F_{v}$, whence $F_{v}^{p^{-m}}=F^{p^{-m}} F_{v}$. Now let $a \in \bar{K}$ be inseparable over $K$. Then there exists an integer $m \geq 1$ and an extension $F / K$ as above such that $K(a)=F^{p^{-m}}$. Consequently, $a \in K(a) \cdot F_{v}=F^{p^{-m}} F_{v}=F_{v}^{p^{-m}}$, whence $a$ is also inseparable over $K_{v}$. This shows that $K^{\mathrm{s}}=\bar{K} \cap K_{v}^{\mathrm{s}}$. Now let $V \subset \mathbb{A}_{K}^{n}$ be an affine $K$-variety and let $P=\left(x_{1}, \ldots, x_{n}\right) \in V(\bar{K}) \cap V\left(K_{v}^{\mathrm{s}}\right)$. Then each $x_{i} \in \bar{K} \cap K_{v}^{\mathrm{s}}=K^{\mathrm{s}}$, whence $P \in V\left(K^{\mathrm{s}}\right)$. Thus $V\left(K^{\mathrm{s}}\right)=V(\bar{K}) \cap V\left(K_{v}^{\mathrm{s}}\right)$, and the lemma is now clear since $A$ is covered by affine $K$-varieties.

If $v$ is a prime of $K$, we will write $\psi_{v}=\psi \times_{\operatorname{Spec} K} \operatorname{Spec} K_{v}$. Since $H^{1}\left(K^{\mathrm{s}}, A\right)=$ $H^{1}\left(K_{v}^{\mathrm{s}}, A\right)=0$, the exact sequence (1) yields a commutative diagram

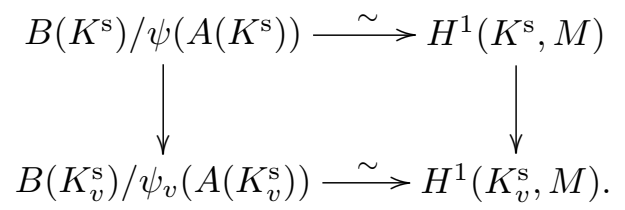

Lemma 2.2. Let $v$ be a prime of $K$. Then the canonical map

$$
B\left(K^{\mathrm{s}}\right) / \psi\left(A\left(K^{\mathrm{s}}\right)\right) \rightarrow B\left(K_{v}^{\mathrm{s}}\right) / \psi_{v}\left(A\left(K_{v}^{\mathrm{s}}\right)\right)
$$

is injective. 
Proof. Write $M=\operatorname{Spec} R$, where $R$ is a finite $K$-algebra, and identify $M\left(\bar{K}_{v}\right)$ with $\operatorname{Hom}_{K_{v}}\left(K_{v} \otimes_{K} R, \bar{K}_{v}\right)$. If $s \in M\left(\bar{K}_{v}\right)$, then the image of the composition $\tilde{f}: R \rightarrow$ $K_{v} \otimes_{K} R \stackrel{s}{\rightarrow} \bar{K}_{v}$ is a finite $K$-algebra and so, in fact, a finite field extension of $K$. Consequently, $\tilde{f}$ factors through some $f \in \operatorname{Hom}_{K}(R, \bar{K})=M(\bar{K})$. This implies that $M\left(\bar{K}_{v}\right)=M(\bar{K})$. Now let $P \in B\left(K^{\mathrm{s}}\right) \cap \psi_{v}\left(A\left(K_{v}^{\mathrm{s}}\right)\right) \subseteq B\left(K_{v}^{\mathrm{s}}\right)$ and let $Q \in$ $A\left(K_{v}^{\mathrm{s}}\right)$ be such that $P=\psi_{v}(Q)$. Since $A(\bar{K}) \stackrel{\psi}{\rightarrow} B(\bar{K})$ is surjective, there exists an $R \in A(\bar{K})$ such that $\psi(R)=P$. Then $R-Q \in M\left(\bar{K}_{v}\right)=M(\bar{K})$. This shows that $Q \in A(\bar{K}) \cap A\left(K_{v}^{\mathrm{s}}\right)=A\left(K^{\mathrm{s}}\right)$, by the previous lemma. Thus $P=\psi(Q) \in \psi\left(A\left(K^{\mathrm{s}}\right)\right)$, as desired.

The above lemma and diagram (2) show that the localization map $H^{1}\left(K^{\mathrm{s}}, M\right) \rightarrow$ $H^{1}\left(K_{v}^{\mathrm{s}}, M\right)$ is injective. The main theorem is now immediate from the exact commutative diagram

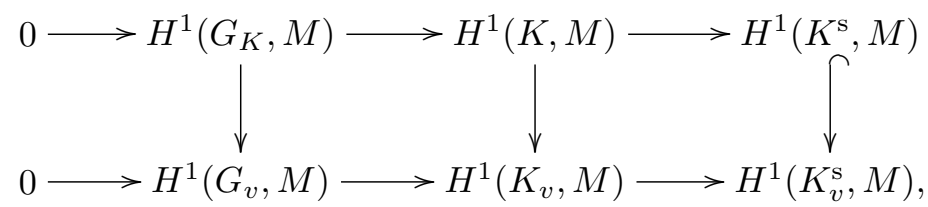

whose rows are the inflation-restriction exact sequences in flat cohomology [16, p.422, line -12].

\section{Applications}

Let $K$ and $M$ be as in the previous section. We will write $K(M)$ for the subfield of $K^{\mathrm{s}}$ fixed by $\operatorname{Ker}\left[G_{K} \rightarrow \operatorname{Aut}\left(M\left(K^{\mathrm{s}}\right)\right)\right]$. We note that the Hasse principle is known to hold for $M\left(K^{\mathrm{s}}\right)$ under any of the following hypotheses:

(a) $\operatorname{Gal}(K(M) / K) \subseteq \operatorname{Aut}\left(M\left(K^{\mathrm{s}}\right)\right)$ is cyclic. See [9, Lemma I.9.3, p.118].

(b) $M\left(K^{\mathrm{s}}\right)$ is a simple $G_{K}$-module such that $p M\left(K^{\mathrm{s}}\right)=0$ and $\operatorname{Gal}(K(M) / K)$ is a $p$-solvable group, i.e., $\operatorname{Gal}(K(M) / K)$ has a composition series whose factors of order divisible by $p$ are cyclic. See [9, Theorem I.9.2(a), p.117].

(c) There exists a set $T$ of primes of $K$, containing the set $S$ of all primes of $K$ which split completely in $K(M)$, such that $T \backslash S$ has Dirichlet density zero and $[K(M): K]=$ l.c.m. $\left\{\left[K(M)_{v}: K_{v}\right]: v \in T\right\}$. See [11, Theorem 9.1.9(iii), p.528].

In this section we focus on case (a) above when $M=A_{p^{m}}$ is the $p^{m}$-torsion subgroup scheme of an abelian variety $A$ defined over $K$. More precisely, we are interested in the class of abelian varieties $A$ such that $A_{p^{m}}\left(K^{\mathrm{s}}\right)$ is cyclic, for then $\operatorname{Gal}\left(K\left(A_{p^{m}}\right) / K\right) \hookrightarrow \operatorname{Aut}\left(A_{p^{m}}\left(K^{\mathrm{s}}\right)\right)$ is cyclic as well if $p$ is odd or $m \leq 2$ and (a) applies. Clearly, this class contains all ordinary abelian varieties $A$ such that the associated Kodaira-Spencer map has maximal rank since, as noted in Section $1, A_{p^{m}}\left(K^{\mathrm{s}}\right)$ is in fact zero. To find more examples, recall that $A_{p^{m}}(\bar{K}) \simeq\left(\mathbb{Z} / p^{m} \mathbb{Z}\right)^{f}$ for some integer $f$ (called the $p$-rank of $A$ ) such that $0 \leq f \leq \operatorname{dim} A$. Thus, if $f \leq 1$, then $A_{p^{m}}\left(K^{\mathrm{s}}\right)$ is cyclic. Clearly, the condition $f \leq 1$ holds if $A$ is an elliptic curve, but there exist higher-dimensional abelian varieties $A$ for which $f \leq 1$. See [13, Section 4].

Remark 3.1. Clearly, $\operatorname{Gal}\left(K\left(A_{p^{m}}\right) / K\right)$ may be cyclic even if $A_{p^{m}}\left(K^{\mathrm{s}}\right)$ is not. For example, let $k$ be the (finite) field of constants of $K$, let $A_{0}$ be an abelian variety 
defined over $k$ and let $A=A_{0} \times_{\text {Spec } k}$ Spec $K$ be the constant abelian variety over $K$ defined by $A_{0}$. Then $A\left(K^{\mathrm{s}}\right)_{\text {tors }}=A_{0}(\bar{k})$, and therefore $\operatorname{Gal}\left(K\left(A_{p^{m}}\right) / K\right) \simeq \operatorname{Gal}\left(k^{\prime} / k\right)$ for some finite extension $k^{\prime}$ of $k$. Consequently, $\operatorname{Gal}\left(K\left(A_{p^{m}}\right) / K\right)$ is cyclic and the Hasse principle holds for $A_{p^{m}}\left(K^{\mathrm{s}}\right)$.

We will write $A\{p\}$ for the $p$-divisible group attached to $A$, i.e., $A\{p\}=\lim _{\longrightarrow} A_{p^{m}}$. If $B$ is an abelian group, $B(p)=\cup_{m} B_{p^{m}}$ is the $p$-primary component of its torsion subgroup, $B^{\wedge}=\lim _{m} B / p^{m}$ is the $p$-adic completion of $B$ and $T_{p} B=\varliminf_{m} B_{p^{m}}$ is the $p$-adic Tate module of $B$. Further, if $B$ is a topological abelian group, $B^{D}$ will denote $\operatorname{Hom}_{\text {cont. }}(B, \mathbb{Q} / \mathbb{Z})$ endowed with the compact-open topology, where $\mathbb{Q} / \mathbb{Z}$ is given the discrete topology.

Let $X$ denote the unique smooth, projective and irreducible curve over the field of constants of $K$ having function field $K$. If $A$ is an abelian variety over $K$, we will write $\mathscr{A}$ for the Néron model of $A$ over $X$.

The following statement is immediate from the main theorem and the above remarks.

Proposition 3.2. Let $A$ be an abelian variety defined over $K$ and let $m$ be a positive integer. Assume that $A_{p^{m}}\left(K^{\mathrm{s}}\right)$ is cyclic. Assume, in addition, that $m \leq 2$ if $p=2$. Then the localization map in flat cohomology

$$
H^{1}\left(K, A_{p^{m}}\right) \rightarrow \prod_{\text {all } v} H^{1}\left(K_{v}, A_{p^{m}}\right)
$$

is injective.

The next lemma confirms a long-standing and widely-held expectation.

Lemma 3.3. $H^{2}(K, A)=0$.

Proof. Since $H^{2}\left(K_{v}, A\right)=0$ for every $v$ [9, Theorem III.7.8, p.285], it suffices to check that $\amalg^{2}(A)=0$. For any integer $n$, there exists a canonical exact sequence of flat cohomology groups

$$
0 \rightarrow H^{1}(K, A) / n \rightarrow H^{2}\left(K, A_{n}\right) \rightarrow H^{2}(K, A)_{n} \rightarrow 0 .
$$

Since the Galois cohomology groups $H^{i}(K, A)$ are torsion in degrees $i \geq 1$ and $\mathbb{Q} / \mathbb{Z}$ is divisible, the direct limit over $n$ of the above exact sequences yields a canonical isomorphism $H^{2}(K, A)=\lim _{n} H^{2}\left(K, A_{n}\right)$. An analogous isomorphism exists over $K_{v}$ for each prime $v$ of $K$, and we conclude that $\amalg^{2}(A)$ is canonically isomorphic to $\lim _{\longrightarrow} \Psi^{2}\left(A_{n}\right)$. Now, by Poitou-Tate duality [9, Theorem I.4.10(a), p.57] and [4, Theorem 4.8], the latter group is canonically isomorphic to the Pontryagin dual of $\lim _{n} \amalg^{1}\left(A_{n}^{t}\right)$, where $A^{t}$ is the dual abelian variety of $A$. Thus, it suffices to show that $\varlimsup_{n} \amalg^{1}\left(A_{n}^{t}\right)=0$. Let $U$ be the largest open subscheme of $X$ such that $A_{n}^{t}$ extends to $\overleftarrow{n}_{n}$ a fite and flat $U$-group scheme $\mathscr{A}_{n}^{t}$. For each closed point $v$ of $U$, let $\mathcal{O}_{v}$ denote the completion of the local ring of $U$ at $v$. Now let $V$ be any non-empty open subscheme of $U$. By the computations at the beginning of [9, III.7, p.280] and the localization sequence [8, Proposition III.1.25, p.92], there exists an exact sequence

$$
0 \rightarrow H^{1}\left(U, \mathscr{A}_{n}^{t}\right) \rightarrow H^{1}\left(V, \mathscr{A}_{n}^{t}\right) \rightarrow \bigoplus_{v \in U \backslash V} H^{1}\left(K_{v}, A_{n}^{t}\right) / H^{1}\left(\mathcal{O}_{v}, \mathscr{A}_{n}^{t}\right)
$$


Taking the direct limit over $V$ in the above sequence and using [4, Lemma 2.3], we obtain an exact sequence

$$
0 \rightarrow H^{1}\left(U, \mathscr{A}_{n}^{t}\right) \rightarrow H^{1}\left(K, A_{n}^{t}\right) \rightarrow \prod_{v \in U} H^{1}\left(K_{v}, A_{n}^{t}\right) / H^{1}\left(\mathcal{O}_{v}, \mathscr{A}_{n}^{t}\right)
$$

where the product extends over all closed points of $U$. The exactness of the last sequence shows the injectivity of the right-hand vertical map in the diagram below

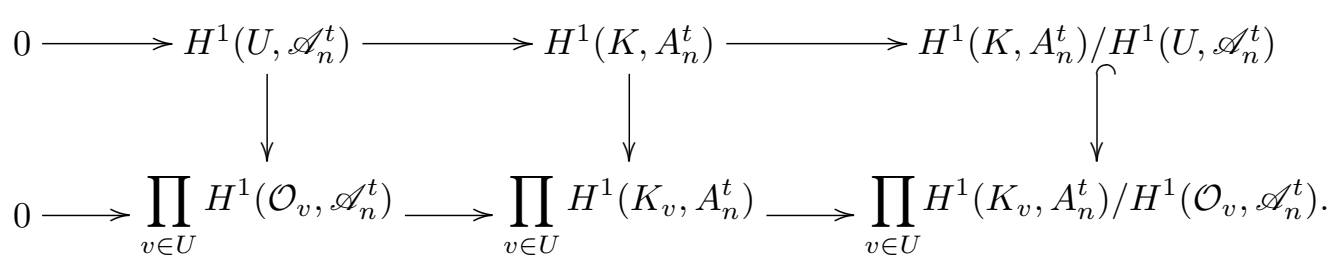

We conclude that $\amalg_{U}^{1}\left(A_{n}^{t}\right):=\operatorname{Ker}\left[H^{1}\left(K, A_{n}^{t}\right) \rightarrow \prod_{v \in U} H^{1}\left(K_{v}, A_{n}^{t}\right)\right]$ equals

$$
\bar{H}^{1}\left(U, \mathscr{A}_{n}^{t}\right):=\operatorname{Ker}\left[H^{1}\left(U, \mathscr{A}_{n}^{t}\right) \rightarrow \prod_{v \in U} H^{1}\left(\mathcal{O}_{v}, \mathscr{A}_{n}^{t}\right)\right] .
$$

Now, it is shown in [10, Propositions 5 and 6], that $\lim _{n} \bar{H}^{1}\left(U, \mathscr{A}_{n}^{t}\right)=0$, whence $\varliminf_{n} \amalg_{U}^{1}\left(A_{n}^{t}\right)=0$. Now the exact sequence

$$
0 \rightarrow \amalg^{1}\left(A_{n}^{t}\right) \rightarrow \amalg_{U}^{1}\left(A_{n}^{t}\right) \rightarrow \prod_{v \notin U} H^{1}\left(K_{v}, A_{n}^{t}\right)
$$

shows that $\lim _{n} \amalg^{1}\left(A_{n}^{t}\right)=0$, as desired.

Remark 3.4. With the notation of the above proof, the kernel-cokernel exact sequence [9, Proposition I.0.24, p.16] for the pair of maps

$$
H^{1}\left(K, A_{n}^{t}\right) \rightarrow \bigoplus_{\text {all } v} H^{1}\left(K_{v}, A_{n}^{t}\right) \rightarrow \bigoplus_{\text {all } v} H^{1}\left(K_{v}, A^{t}\right)
$$

yields an exact sequence

$$
0 \rightarrow \amalg^{1}\left(A_{n}^{t}\right) \rightarrow \operatorname{Sel}\left(A^{t}\right)_{n} \rightarrow \bigoplus_{\text {all } v} H^{0}\left(K_{v}, A^{t}\right) / n,
$$

where $\operatorname{Sel}\left(A^{t}\right)_{n}:=\operatorname{Ker}\left[H^{1}\left(K, A_{n}^{t}\right) \rightarrow \oplus_{\text {all }} v H^{1}\left(K_{v}, A^{t}\right)\right]$. Since ${\underset{\varliminf}{\longleftarrow}}_{n} \amalg^{1}\left(A_{n}^{t}\right)=0$ as shown above, the inverse limit over $n$ of the preceding sequences yields an injection

$$
\lim _{n} \operatorname{Sel}\left(A^{t}\right)_{n} \hookrightarrow \prod_{\text {all } v} \lim _{n} H^{0}\left(K_{v}, A^{t}\right) / n
$$

This injectivity was claimed in [3, p.300, line -8], but the "proof" given there is inadequate and should be replaced by the above one.

Proposition 3.5. Let $A$ be an abelian variety over $K$ and let $A^{t}$ be the corresponding dual abelian variety. Assume that $A_{p^{m}}^{t}\left(K^{\mathrm{s}}\right)$ is cyclic, where $m$ is a positive integer such that $m \leq 2$ if $p=2$. Then the localization maps

$$
H^{2}\left(K, A_{p^{m}}\right) \rightarrow \bigoplus_{\text {all } v} H^{2}\left(K_{v}, A_{p^{m}}\right)
$$


and

$$
H^{1}(K, A) / p^{m} \rightarrow \bigoplus_{\text {all } v} H^{1}\left(K_{v}, A\right) / p^{m}
$$

are injective.

Proof. The injectivity of the first map is immediate from Proposition 3.2 and PoitouTate duality [4, Theorem 4.8]. On the other hand, the lemma and the long exact flat cohomology sequence associated to $0 \rightarrow A_{p^{m}} \rightarrow A \stackrel{p^{m}}{\rightarrow} A \rightarrow 0$ over $K$ and over $K_{v}$ for each $v$ identifies the second map of the statement with the first, thereby completing the proof.

Remark 3.6. When $A$ is an elliptic curve over a number field and $p$ is any prime, the injectivity of the second map in the above proposition was first established in $[2$, Lemma 6.1, p.107].

Let $\prod_{\text {all } v}^{\prime} H^{1}\left(K_{v}, A_{p^{m}}\right)$ denote the restricted product of the groups $H^{1}\left(K_{v}, A_{p^{m}}\right)$ with respect to the subgroups $H^{1}\left(\mathcal{O}_{v}, \mathscr{A}_{p^{m}}\right)$.

Proposition 3.7. Let $A$ be an abelian variety over $K$ and let $m$ be a positive integer such that $m \leq 2$ if $p=2$. Assume that both $A_{p^{m}}\left(K^{\mathrm{s}}\right)$ and $A_{p^{m}}^{t}\left(K^{\mathrm{s}}\right)$ are cyclic. Then there exist exact sequences

$$
\begin{gathered}
0 \rightarrow A_{p^{m}}(K) \rightarrow \prod_{\text {all } v} A_{p^{m}}\left(K_{v}\right) \rightarrow H^{2}\left(K, A_{p^{m}}^{t}\right)^{D} \rightarrow 0, \\
0 \rightarrow H^{1}\left(K, A_{p^{m}}\right) \rightarrow \prod_{\text {all } v}^{\prime} H^{1}\left(K_{v}, A_{p^{m}}\right) \rightarrow H^{1}\left(K, A_{p^{m}}^{t}\right)^{D} \rightarrow 0
\end{gathered}
$$

and

$$
0 \rightarrow H^{2}\left(K, A_{p^{m}}\right) \rightarrow \bigoplus_{\text {all } v} H^{2}\left(K_{v}, A_{p^{m}}\right) \rightarrow A_{p^{m}}^{t}(K) \rightarrow 0 .
$$

Proof. This is immediate from Propositions 3.2 and 3.5 and the Poitou-Tate exact sequence in flat-cohomology [4, Theorem 4.11].

Now set

$$
\operatorname{Sel}(A)_{p^{m}}=\operatorname{Ker}\left[H^{1}\left(K, A_{p^{m}}\right) \rightarrow \bigoplus_{\text {all } v} H^{1}\left(K_{v}, A\right)\right]
$$

and define $T_{p} \operatorname{Sel}(A)=\varliminf_{m} \operatorname{Sel}(A)_{p^{m}}$. Further, recall the group

$$
\amalg^{1}(A)=\operatorname{Ker}\left[H^{1}(K, A) \rightarrow \bigoplus_{\text {all } v} H^{1}\left(K_{v}, A\right)\right] .
$$

Further, set $H^{1}\left(K, T_{p} A^{t}\right)=\varliminf_{m} H^{1}\left(K, A_{p^{m}}^{t}\right)$.

Corollary 3.8. Under the hypotheses of the proposition, there exist canonical exact sequences

$$
\begin{gathered}
0 \rightarrow \amalg^{1}(A)(p) \rightarrow \frac{\prod_{\text {all } v} A\left(K_{v}\right) \otimes \mathbb{Q}_{p} / \mathbb{Z}_{p}}{A(K) \otimes \mathbb{Q}_{p} / \mathbb{Z}_{p}} \rightarrow H^{1}\left(K, T_{p} A^{t}\right)^{D} \rightarrow\left(T_{p} \operatorname{Sel}\left(A^{t}\right)\right)^{D} \rightarrow 0, \\
0 \rightarrow T_{p} \amalg^{1}(A) \rightarrow\left(\prod_{\text {all } v} A\left(K_{v}\right)^{\wedge}\right) / A(K)^{\wedge} \rightarrow H^{1}\left(K, A^{t}\{p\}\right)^{D}
\end{gathered}
$$


and

$$
0 \rightarrow T_{p} \operatorname{Sel}(A) \rightarrow \prod_{\text {all } v} A\left(K_{v}\right)^{\wedge} \rightarrow H^{1}\left(K, A^{t}\{p\}\right)^{D} .
$$

Proof. Let $m \geq 1$ be an integer. The exact commutative diagram

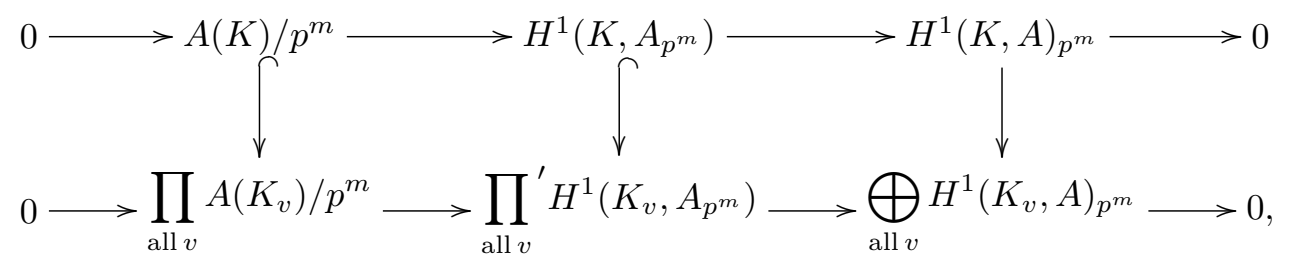

yields an exact sequence of profinite abelian groups

$$
0 \rightarrow \amalg^{1}(A)_{p^{m}} \rightarrow \frac{\prod_{\mathrm{all} v} A\left(K_{v}\right) / p^{m}}{A(K) / p^{m}} \rightarrow H^{1}\left(K, A_{p^{m}}^{t}\right)^{D} \rightarrow \mathrm{Б}_{m}(A) \rightarrow 0,
$$

where $\mathrm{5}_{m}(A)=$ Coker $\left[H^{1}(K, A)_{p^{m}} \rightarrow \oplus_{\text {all } v} H^{1}\left(K_{v}, A\right)_{p^{m}}\right]$. By the main theorem of [3], $\lim _{\longrightarrow} \mathrm{S}_{m}(A) \simeq\left(T_{p} \operatorname{Sel}\left(A^{t}\right)\right)^{D}$ and the first exact sequence of the statement follows by taking the direct limit over $m$ in (3). On the other hand, since the inverse limit functor is exact on the category of profinite groups [15, Proposition 2.2.4, p. 32], the inverse limit over $m$ of the sequences (3) is the second exact sequence of the statement. The third exact sequence follows from the second and the exact commutative diagram

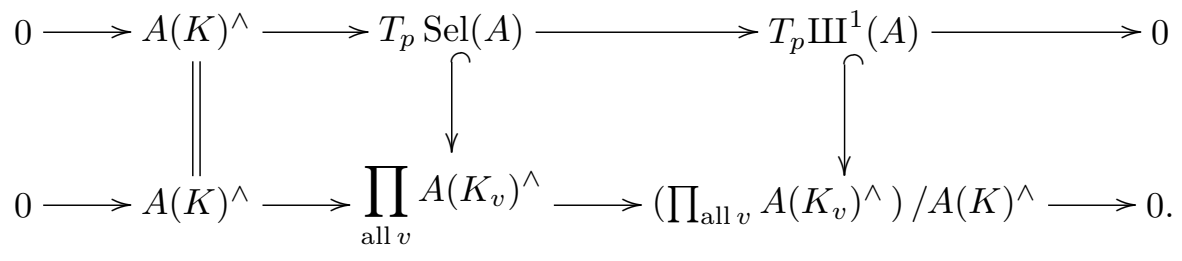

\section{Concluding remarks}

Let $K$ and $M$ be as in Section 1 and assume that $M$ has $p$-power order. Further, let $M^{*}$ denote the Cartier dual of $M$. Since $\amalg^{1}\left(K, M^{*}\right) \simeq \amalg^{1}\left(G_{K}, M^{*}\right)$ and there exists a perfect pairing of finite groups

$$
\amalg^{1}\left(K, M^{*}\right) \times \amalg^{2}(K, M) \rightarrow \mathbb{Q} / \mathbb{Z}
$$

by [4], Theorem 4.8, it is natural to expect a Galois-cohomological description of $\amalg^{2}(K, M)$. Note, however, that the natural guess $\amalg^{2}(K, M) \simeq \amalg^{2}\left(G_{K}, M\right)$ is incorrect since the latter group is zero (because the $p$-cohomological dimension of $G_{K}$ is $\left.\leq 1\right)$. To obtain the correct answer, we proceed as follows. Since $H^{i}\left(K^{\mathrm{s}}, A\right)=$ $H^{i}\left(K^{\mathrm{s}}, B\right)=0$ for all $i \geq 1$, the exact sequence (1) shows that $H^{i}\left(K^{\mathrm{s}}, M\right)=0$ for all $i \geq 2$. On the other hand, $H^{i}\left(G_{K}, M\right)=0$ for all $i \geq 2$ as well, since $\operatorname{cd}_{p}\left(G_{K}\right) \leq 1$. Now the exact sequence of terms of low degree belonging to the Hochschild-Serre spectral sequence $H^{i}\left(G_{K}, H^{j}\left(K^{\mathrm{s}}, M\right)\right) \Rightarrow H^{i+j}(K, M)$ yields a canonical isomorphism

$$
H^{2}(K, M) \simeq H^{1}\left(G_{K}, H^{1}\left(K^{\mathrm{s}}, M\right)\right) \simeq H^{1}\left(G_{K}, B\left(K^{\mathrm{s}}\right) / \psi\left(A\left(K^{\mathrm{s}}\right)\right)\right)
$$


Analogous isomorphisms exist over $K_{v}$ for every prime $v$ of $K$, and we conclude that

$$
\amalg^{2}(K, M) \simeq \amalg^{1}\left(G_{K}, B / \psi(A)\right) .
$$

For example, if $M=A_{p^{m}}$ for an abelian variety $A$ over $K$, then $\amalg^{2}\left(K, A_{p^{m}}\right) \simeq \amalg^{1}$ $\left(G_{K}, A / p^{m}\right)$ and the pairing (4) takes the form

$$
\amalg^{1}\left(G_{K}, A_{p^{m}}^{t}\right) \times \amalg^{1}\left(G_{K}, A / p^{m}\right) \rightarrow \mathbb{Q} / \mathbb{Z} .
$$

In particular, if $A_{p^{m}}^{t}\left(K^{\mathrm{s}}\right)$ is cyclic with $m \leq 2$ if $p=2$, then Proposition 3.2 applied to $A^{t}$ and the perfectness of the above pairing yield $\amalg^{1}\left(G_{K}, A / p^{m}\right)=0$, i.e., the Hasse principle holds for the $G_{K}$-module $A\left(K^{\mathrm{s}}\right) / p^{m}$.

\section{Acknowledgments}

C.G.-A. is partially supported by Fondecyt grant no. 1080025. K.-S. T. is partially supported by the National Science Council of Taiwan, NSC99-2115-M-002-002-MY3.

\section{References}

[1] P. Berthelot, L. Breen and W. Messing, Théorie de Dieudonné Cristalline II, Lect. Notes Math. 930, Springer-Verlag, 1982.

[2] J. W. S. Cassels, Arithmetic on curves of genus 1, IV. Proof of the Hauptvermutung, J. Reine Angew. Math. 211 (1962), 95-112.

[3] C. D. González-Avilés and K.-S. Tan, A generalization of the Cassels-Tate dual exact sequence, Math. Res. Lett. 14(2) (2007), 295-302.

[4] C. D. González-Avilés, Arithmetic duality theorems for 1-motives over function fields, J. Reine Angew. Math. 632 (2009), 203-231.

[5] A. Grothendieck, Le groupe de Brauer III. In Dix Exposées sur la Cohomologie des Schémas, North-Holland, Amsterdam, 1968, 88-188.

[6] U. Jannsen, Galoismoduln mit Hasse-Prinzip, J. Reine Angew. Math. 337 (1982), 154-158.

[7] J. S. Milne, Elements of order $p$ in the Tate-Shafarevic group, Bull. Lond. Math. Soc. 2 (1970), 293-296.

[8] J. S. Milne, Étale Cohomology, Princeton University press, Princeton, 1980.

[9] J. S. Milne, Arithmetic duality theorems, 2nd ed. (electronic version), 2006.

[10] J. S. Milne, Congruence subgroups of abelian varieties, Bull. Sci. Math. 96 (1972), 333-338.

[11] J. Neukirch, A. Schmidt and K. Wingberg, Cohomology of number fields, 2nd ed. Springer, 2008.

[12] O. Neumann, Einige klassen von endlichen Galois-moduln mit Hasse-prinzip, Math. Nachr. 72 (1976), 305-320.

[13] R. Pries, A short guide to p-torsion of abelian varieties in characteristic p, Computational arithmetic geometry, 121129, Contemp. Math. 463, Amer. Math. Soc., Providence, RI, 2008.

[14] M. Raynaud, "p-torsion" du schema de Picard, Journal de Géométrie Algébrique de Rennes (Rennes, 1978), Vol. II, Astérisque 64 (1979), 87-148.

[15] L. Ribes and P. Zalesskii: Profinite groups, Ergeb. Math. Ser. 40, Springer, Berlin, 2000.

[16] S. Shatz, Cohomology of artinian group schemes over local fields, Ann. Math. 79(3) (1964), 411-449.

[17] J. F. Voloch, Diophantine approximation on abelian varieties in characteristic $p$, Amer. J. Math. 117 (1995), 1089-1095.

Departamento de Matemáticas, Universidad de la Serena, Chile

E-mail address: cgonzalez@userena.cl

Department of Mathematics, National Taiwan University, Taipei 10764, Taiwan

E-mail address: tan@math.ntu.edu.tw 